\title{
Jejunal Hemorrhage, CTCAE
}

National Cancer Institute

\section{Source}

National Cancer Institute. Jejunal Hemorrhage, CT CAE. NCI Thesaurus. Code C56543.

A disorder characterized by bleeding from the jejunal wall. 